\title{
EVALUACIÓN DE LA FERTILIZACIÓN NITROGENADA EN ARROZ INUNDADO ${ }^{1}$
}

\author{
Rodolfo Quirós-Herrera², Carlos Ramírez-Martínez ${ }^{3}$
}

\begin{abstract}
RESUMEN
Evaluación de la fertilización nitrogenada en arroz inundado. Para mejorar los rendimientos y propiciar un manejo sostenible del arroz es necesario aumentar la eficiencia de uso del N por el cultivo. Con ese enfoque en el año 2003 se realizó un experimento de campo para determinar si el cultivo de arroz inundado con el sistema siembra directa sobre rastrojos (SDR) necesita aplicar dosis mayores de $\mathrm{N}$ en comparación con lo que se aplica en labranza mecanizada convencional (LMC). Se compararon los rendimientos del arroz según distintas combinaciones de dosis y épocas de fertilización con $\mathrm{N}$, en un lote con suelos vertisoles. El sitio corresponde a la zona de vida bosque húmedo premontano transición a basal tropical, en la Región Pacífico Norte de Costa Rica. Tanto en LMC como en SDR se determinó que la fertilización del arroz CR-1113, con $120 \mathrm{~kg} / \mathrm{ha}$ de N fraccionados en tres aplicaciones permitió mayores rendimientos a los alcanzados con la dosis completa en una sola aplicación temprana. No se encontró suficiente evidencia para justificar que en SDR se fertilice con mayor cantidad de N que en LMC. El instrumento medidor de clorofila (IMC) fue una herramienta de campo efectivo para diagnosticar el contenido de $\mathrm{N}$ foliar en arroz, en forma rápida y económica. Sin embargo, su uso no permitió alcanzar la precisión que se logra mediante análisis foliares de laboratorio.
\end{abstract}

Palabras clave: Arroz inundado, fertilización nitrogenada, siembra directa, medidor de clorofila.

\begin{abstract}
Evaluation of nitrogen fertilization in a flooded rice field. To improve yields and facilitate a sustainable management of rice it is necessary to increase the $\mathrm{N}$ use efficiency of the crop. From this point of view, a field experiment was carried out during 2003 to determine if higher $\mathrm{N}$ fertilization rates must be applied in a flooded rice crop using direct seeding into stubble (DSS) in comparison with conventional mechanized tillage (CMT). Rice crop yields were compared using different combinations of rates and timing of $\mathrm{N}$ fertilization, in a field with Vertisol soils. The site belongs to the humid pre mountain forest transition to tropical basal life zone in the North Pacific Region of Costa Rica. In CMT as well as in DSS it was determined that CR-1113 rice fertilization with $120 \mathrm{~kg} / \mathrm{ha}$ of $\mathrm{N}$ split in three applications allows higher yields than those obtained with an early single dose. Enough evidence to justify a higher fertilization in DSS than in CMT was not found. It was shown that chlorophyll-measuring device (SPAD) is a practical tool to diagnose foliar $\mathrm{N}$ content in rice, in a rapid and economic way. However, its use does not allow reaching the accuracy obtained through laboratory foliar analysis.
\end{abstract}

Key words: Flooded rice, nitrogen fertilization, No-till, chlorophyll meter SPAD.

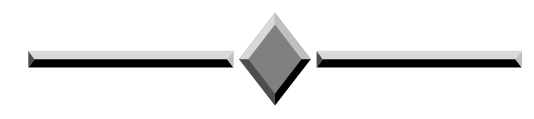

\section{INTRODUCCIÓN}

$\mathrm{Al}$ igual que en otros cultivos, el nitrógeno $(\mathrm{N})$ es el principal factor limitante en la producción agrícola del arroz (Oryza sativa L.). Su disponibilidad se considera esencial por ser un componente básico en todas las moléculas orgánicas involucradas en el crecimiento y desarrollo vegetal (Salas 2003). Las dos formas

1 Recibido: 28 de marzo, 2005. Aceptado: 17 de julio, 2006. Parte de la tesis del primer autor, Programa de Doctorado en sistemas de producción agrícola tropical sostenible "SPATS", Universidad de Costa Rica.

2 Estudiante programa de doctorado en SPATS, Facultad de Ciencias Agroalimentarias, Universidad de Costa Rica. Correo electrónico: rodolfo.quiros@costarricense.cr

3 Correo electrónico: carlosm20@gmail.com 
como el $\mathrm{N}$ puede ser absorbido por las plantas son amonio $\left(\mathrm{NH}_{4}{ }^{+}\right)$y nitrato $\left(\mathrm{NO}_{3}{ }^{-}\right)$, principalmente obtenidas de los fertilizantes nitrogenados y la mineralización de los residuos de cosecha y la materia orgánica del suelo. En mayor proporción que en otros cultivos, la productividad del arroz depende de la disponibilidad y eficiencia en la absorción del $\mathrm{N}$, tanto por su contribución directa como por permitir la absorción de otros nutrimentos (Norman et al. 2003; Ramírez 2001).

En los campos de arroz inundado existen múltiples factores que ocasionan pérdidas del $\mathrm{N}$ y causan baja respuesta del cultivo a la fertilización nitrogenada (De Datta 1981). Aunque el cultivo es capaz de usar en forma efectiva las fuentes amoniacales, cuando se aplica una dosis más alta de la necesaria o su distribución es inapropiada, ocurren pérdidas especialmente por desnitrificación y volatilización (David y Otsuka 1994). Cuando se aplica urea sobre la lámina de agua o sobre barro, las pérdidas de $\mathrm{N}$ por volatilización pueden ser hasta del 80\% (De Datta 1981). Por otra parte, si el N se aplica en forma de nitratos, podrían ocurrir pérdidas por lixiviación, y por tal razón no debe utilizarse tales fuentes de $\mathrm{N}$ en agroecosistemas inundados (Molina 2003).

Según algunos autores (Wilson et al. 1998; Jaramillo et al. 2003), una forma de minimizar las pérdidas por volatilización es fertilizar sobre suelo seco, con la dosis completa de $\mathrm{N}$ en una sola aplicación temprana, justo antes de introducir la lámina de agua permanente. Esto es posible gracias a que la adsorción del amonio a las arcillas es mayor en el suelo seco que en el suelo húmedo, pues el mojado del suelo reduce la superficie de contacto directo con las arcillas. Además, aplicando una lámina de agua inmediatamente después de la fertilización se induce la anaerobiosis, se inhibe la nitrificación y en consecuencia disminuyen las pérdidas de $\mathrm{N}$ por volatilización (Molina 2003). Sin embargo en la práctica los productores por lo general fraccionan los fertilizantes nitrogenados en tres o más aplicaciones de acuerdo al desarrollo fenológico del cultivo.

La dosis y la época óptima de fertilización nitrogenada para el cultivo de arroz puede variar ampliamente según las condiciones de clima y suelo (Rico y De Datta 1982). Con base en investigaciones y pruebas de campo realizadas hace más de diez años, fueron establecidas las estrategias de fertilización apropiadas para siembras convencionales de arroz empleadas actualmente en el país (Coto-Gaucherand 1991; Cordero 1993). Sin embargo, el estudio de la fertilización en sistemas de labranza reducida, tales como la siembra directa sobre rastrojos (SDR) no ha recibido la misma importancia (Rojas et al. 2002). Este sistema se caracteriza por dejar sobre el terreno todos los rastrojos del ciclo de cultivo anterior y no efectuar operaciones de labranza del suelo. En SDR la eficiencia en la fertilización nitrogenada del arroz puede disminuir con respecto a la lograda en sistemas convencionales, debido a la influencia de los rastrojos y a la dificultad para incorporar el $\mathrm{N}$ dentro de un suelo que no ha sido disturbado por la labranza.

Los rastrojos acumulados en la superficie pueden modificar la disponibilidad de $\mathrm{N}$ para el cultivo porque en su descomposición por los microorganismos del suelo se consume parte de las existencias disponibles. Esto podría ser perjudicial al cultivo, especialmente en los suelos vertisoles comunes de las áreas arroceras del Pacífico Norte, cuyas arcillas con cargas negativas tienen alta capacidad para adsorber el amonio (Molina 2003). Puesto que la SDR afecta de distinta manera la dinámica del $\mathrm{N}$ en el suelo, los valores óptimos de fertilización podrían ser diferentes a los recomendados para cultivos en labranza mecanizada convencional (LMC). Bird et al. (2001) encontraron que el $\mathrm{N}$ de la fertilización, inmovilizado por los microorganismos en la descomposición de rastrojos, con el tiempo constituyó una fuente que suplió en parte la demanda de $\mathrm{N}$ del cultivo.

Algunas propiedades físicas del suelo cambian a medida que transcurren más ciclos en SDR. Los rastrojos progresivamente se van acumulando sobre la superficie del terreno, contribuyendo a reducir la erosión y la pérdida de agua del suelo por evaporación (Eck y Jones 1992). Además incrementan la retención de humedad a niveles favorables para la germinación y el crecimiento del cultivo. A largo plazo en el perfil de suelos sin labrar la estructura es más estable, con mayor número de macroporos y canales creados por lombrices, propiciando mayores tasas de infiltración con respecto a suelos en LMC (Edwards et al. 1998). Esos cambios en el ambiente edáfico afectan la dinámica del $\mathrm{N}$ en el suelo.

A sólo diez días después de germinadas las plántulas de arroz son capaces de absorber nutrientes de la solución del suelo y empezar el crecimiento vegetativo con mayores posibilidades de generar altos rendimientos 
(De Datta 1981). El N en forma amónica que el arroz absorbe activamente en etapas tempranas se aprovecha en la síntesis de proteínas, producción de macollas y vainas de las hojas (Yoshida 1981). De lo anterior se desprende que a mayor absorción de $\mathrm{N}$ mayor cantidad de macollas efectivas por unidad de superficie con mayor número de tallos fértiles. En consecuencia, el contenido óptimo de $\mathrm{N}$ entre la fase de máximo macollamiento y la formación de panículas permite una adecuada densidad de panículas durante la floración (Tinarelli 1989).

En el estado inicial de formación de panícula la disponibilidad de $\mathrm{N}$ es indispensable para fortalecer el desarrollo reproductivo del arroz. Entre 20 y 25 días antes de la floración la planta de arroz empieza su etapa reproductiva con la formación del primordio de panícula. En ese momento el nudo de la hoja bandera coincide con el de la hoja siguiente y dentro de la vaina la panícula en formación mide entre 1 y $2 \mathrm{~mm}$ de longitud (De Datta 1981). Puesto que en esa fase gran parte de la energía metabólica de la planta es usada para formar espiguillas y granos, con intensa emisión de raíces adventicias superficiales, la absorción de $\mathrm{N}$ se incrementa significativamente. El adecuado abastecimiento con $\mathrm{N}$ en este período asegura mayor cantidad de espiguillas, mayor tamaño de panícula y máxima cantidad de granos llenos por espiguilla (Yoshida 1981; De Datta 1981; Tinarelli 1989).

Conocer el estado nutricional del cultivo en sus diferentes fases fenológicas es indispensable al definir momentos propicios para efectuar la fertilización nitrogenada. El análisis de tejidos verdes (análisis foliares) es una forma de conocer el contenido de $\mathrm{N}$ total y la necesidad de este nutrimento en las plantas de arroz. Aunque esta técnica proporciona resultados precisos, su costo y las demoras necesarias para completar los análisis en laboratorio hacen que sea inadecuada para efectuar recomendaciones de fertilización oportunamente. Un recurso alternativo al análisis foliar es la estimación del contenido de $\mathrm{N}$ foliar, a través de mediciones de clorofila, puesto que facilita la estimación del estatus del $\mathrm{N}$ en las hojas y tallos verdes de la planta en forma inmediata (Peterson et al. 1993). Mientras que la deficiencia de $\mathrm{N}$ se manifiesta con un amarillamiento que comienza en las hojas inferiores más viejas; en plantas adecuadamente fertilizadas con ese elemento se observa un color verde intenso (Salas 2003). Dada esta respuesta fisiológica, se puede inferir el contenido de $\mathrm{N}$ mediante la medición de la clorofila en la planta, conocer de este modo el estado nutricional del cultivo

ISSN: 1021-7444
(Villar y Ortega 2002) y ayudar en la toma de decisiones con respecto a la cantidad y momento propicio de efectuar la fertilización nitrogenada del arrozal.

Varios estudios han mostrado que a través del uso de un instrumento medidor del contenido de clorofila en tejidos verdes $(\mathrm{IMC})^{4}$ se puede estimar el $\mathrm{N}$ presente en las hojas del arroz (Lihong et al. 2004; Chung et al. 2000; Stevens y Hefner 1999). Este instrumento ha sido empleado para predecir las necesidades de $\mathrm{N}$ en diversos cultivos como maíz, trigo, tomate, papa y sorgo (Novoa y Villagrán 2002; Singh et al. 2002; Arregui et al. 2000; Rangel-Lucio et al. 2002). Sin embargo, otros estudios han encontrado limitaciones en el uso práctico de las mediciones de clorofila debido a diferencias en las variedades o a condiciones ambientales cambiantes, tales como: estrés hídrico, temperaturas extremas, deficiencias nutricionales distintas al $\mathrm{N}$, o daños causados por insectos. Por estas razones, para lograr mayor precisión en las estimaciones del instrumento medidor de clorofila (IMC) se requiere determinar la relación particular para cada cultivo y variedad.

El presente estudio compara el efecto de distintas combinaciones de dosis de $\mathrm{N}$ y variantes en el fraccionamiento de las mismas, sobre los rendimientos de un arrozal inundado, tanto en SDR como en LMC. Se evalúa la conveniencia de agregar una cantidad adicional en la fertilización nitrogenada en SDR y se contrasta los rendimientos de una sola aplicación de $\mathrm{N}$ al inicio del cultivo, con respecto a las prácticas convencionales con dosis fraccionadas. Además se evalúa la funcionalidad del IMC para determinar niveles y momentos de fertilización nitrogenada apropiados para incrementar los rendimientos del cultivo y minimizar los posibles impactos negativos al ambiente.

\section{MATERIALES Y MÉTODOS}

\section{Descripción del sitio del experimento}

El experimento fue realizado durante la siembra de verano 2002-2003 en un lote comercial de arroz

\footnotetext{
4 IMC: Por sus siglas en inglés este instrumento para medir el contenido foliar de clorofila es conocido como SPAD-502. El mismo fue desarrollado por la División Especializada en Productos Agrícolas de la Corporación Minolta (Specialty Products Agricultural Division, "SPAD", Minolta Corporation.
}

AGRONOMÍA MESOAMERICANA 17(2): 179-188. 2006 
bajo riego por inundación de la Hacienda El Pelón de la Bajura, en la Región Pacífico Norte de Costa Rica. El sitio había sido cultivado mediante LMC durante doce años consecutivos y con SDR en los últimos tres años. Para instalar el experimento se eligieron terrazas contiguas del lote Playitas con uniformidad en las principales características edáficas. El terreno presentó topografía completamente plana, con una elevación de $20 \mathrm{msnm}$ y había sido previamente micronivelado para manejar una lámina de agua permanente de $10 \mathrm{~cm}$ de altura sobre la superficie del suelo. Durante los meses de la prueba la temperatura media ambiental ${ }^{5}$ fue de 27 ${ }^{\circ} \mathrm{C}$, con valores máximos y mínimos de 35 y $23{ }^{\circ} \mathrm{C}$ respectivamente. Los promedios de las variables radiación solar, humedad relativa y evaporación potencial fueron de $34 \mathrm{~mol} / \mathrm{s}, 85 \%$, y $3,8 \mathrm{~mm} /$ día, en forma respectiva durante el mismo período. La textura del terreno fue francoarcillosa (33\% arena, 29\% limo, 38\% arcilla), pH 6,5, CICE $22,2 \mathrm{cmol}(+) / 1$ y con $2,36 \%$ de contenido de materia orgánica 6 . En el ensayo se usó arroz CR1113 por ser una variedad ampliamente cultivada por los productores arroceros de la región y por presentar buena respuesta a la fertilización con $\mathrm{N}$.

\section{Diseño experimental}

Se utilizó un diseño factorial en parcelas subdivididas compuesto por dos sistemas de labranza, cinco dosis de fertilización nitrogenada $(0,80,100,120$ y $180 \mathrm{~kg} / \mathrm{ha}$ de N) y cuatro variantes en fraccionamiento de dosis. Los sistemas de labranza SDR y LMC fueron asignados a las parcelas mayores, de forma cuadrada y una hectárea de extensión. Las dosis de fertilizante fueron distribuidas en parcelas menores de $20 \times 100 \mathrm{~m}$. Estas últimas fueron subdivididas en cuatro subparcelas de $20 \times 25 \mathrm{~m}$, cada una con cuatro repeticiones, correspondientes al fraccionamiento de la dosis $(5 \times 25 \mathrm{~m})$. Para evitar la contaminación entre tratamientos, el área efectiva evaluada en el estudio se limitó a $1 \mathrm{~m}^{2} /$ subparcela, producto de sumar cuatro lanzamientos del aro metálico, a lo largo del eje central de cada subparcela.

Las variantes en el fraccionamiento de la dosis fueron definidas de acuerdo con el desarrollo fenológico

\footnotetext{
5 Estación hidrometeorológica Melones de Costa Rica, instalada en la zona aledaña al sitio del experimento.

6 Fuente: Laboratorio de suelos del Centro de Investigaciones Agronómicas. Universidad de Costa Rica.
}

del cultivo, de la siguiente forma: A) aplicación única de la dosis completa sobre suelo seco inmediatamente antes de colocar la lámina de agua permanente (LAP), a 25 días después de la siembra (dds), cuando las plántulas de arroz presentaban cuatro hojitas; B) $60 \%$ de la dosis al momento de aplicar la variante A y el $40 \%$ restante en la fase de iniciación del primordio de panícula (IPP), 65 dds; C) $40 \%$ en forma similar a la variante A, $30 \%$ al IPP y $30 \%$ restante en la fase de floración (F), 85 dds; D) $40 \%$ en forma semejante a la variante A, y fracciones del $20 \%$ a 45 dds durante la fase de macollamiento "M", $20 \%$ al IPP y $20 \%$ al IF respectivamente.

\section{Manejo del cultivo}

En las parcelas correspondientes a SDR todos los rastrojos de arroz del ciclo anterior se dejaron sobre el terreno. El suelo sólo fue disturbado en forma mínima a lo largo de los surcos de siembra. El control de las malezas emergidas y la soca ${ }^{7}$ con rebrotes en crecimiento se efectuó con una aplicación de glifosato a razón de $3 \mathrm{~kg} / \mathrm{ha}$ de producto formulado $68 \mathrm{SG}$, en 220 1/ha de agua, distribuida sobre todo el terreno a cultivar. En las parcelas tratadas con LMC la preparación del terreno consistió en un pase de rastra rompedora, otro de rastra afinadora y finalmente se pasó un rodillo compactador. Los rastrojos fueron incorporados en el perfil del suelo y el terreno quedó finamente mullido sin restos visibles de residuos vegetales en la superficie. Para asegurar un adecuado suministro de fósforo, potasio y magnesio, al momento de la siembra se aplicó 50, 125 y $8 \mathrm{~kg} / \mathrm{ha}$ de súper triple fosfato, sulfato de potasio y óxido de magnesio respectivamente. La aplicación de los tratamientos se realizó en forma manual y la fuente de $\mathrm{N}$ usada en el experimento fue urea $(46 \%$ de $\mathrm{N})$.

\section{Rendimiento del cultivo}

Mediante muestreo sistemático se estimó el rendimiento del arroz seco en granza ${ }^{8}$ por tratamiento $(\mathrm{kg} / \mathrm{ha})$. En la evaluación del rendimiento se usó un marco metálico de $0,25 \mathrm{~m}^{2}$, lanzándolo cuatro veces

7 Rebrotes de arroz generado a partir del sistema radicular del ciclo precedente.

8 El término "arroz en granza" corresponde al grano cosechado al cual aún no le ha sido eliminada su cariópside o cascarilla.

AGRONOMÍA MESOAMERICANA 17(2): 179-188. 2006 
por subparcela. El arroz en granza se cosechó cortando las panículas y desgranándolas a mano. A estas estimaciones se les aplicó un factor de corrección para ajustarlas a $13 \%$ de contenido de humedad y $5 \%$ de impurezas. Para determinar la humedad y el porcentaje de impurezas del grano se siguió el protocolo que se emplea en forma rutinaria en la finca del estudio, por diferencia de pesos en condición húmeda de campo y seco en laboratorio. Adicionalmente se determinó la cantidad de panículas de arroz por metro cuadrado, el número de granos llenos por panícula y el porcentaje de vaneamiento. La limpieza, el desgrane, y el recuento de granos llenos o cariópsides vacías se realizó en forma manual y se usó una balanza analítica para las determinaciones del peso. Con los datos resultantes se efectuó el respectivo análisis de varianza.

\section{Relación entre lecturas del IMC y contenido relati- vo de $\mathbf{N}$ foliar}

Antes de cada aplicación fraccionada de $\mathrm{N}$ se estimó el contenido relativo de clorofila en las hojas del arroz mediante el IMC. Cada medición se efectuó en la hoja situada por debajo de la hoja bandera. Se eligió la segunda hoja por la favorable posición de ésta para captar la radiación solar, ser una hoja completamente formada, lo que no siempre se cumple con la hoja bandera, y por mantener plena actividad fotosintética. Se tomó el promedio de cinco lecturas por hoja, en cada oportunidad el sensor del instrumento fue situado al centro de la lámina, entre el borde y la nervadura central, a lo largo de la misma. Se evaluó cuatro plantas por subparcela, a fin de obtener una medición media asociada a cada lanzamiento del marco metálico utilizado en la evaluación de rendimientos.

En una tarea aparte, para calibrar el IMC y determinar la relación entre lecturas de este instrumento y el contenido de $\mathrm{N}$ foliar se seleccionaron hojas con colores que variaron desde tonos verde oscuros hasta cloróticas (tonos amarillentos). Las hojas fueron cortadas en fragmentos de $1 \mathrm{~cm}^{2}$, y finalmente se obtuvieron 800 muestras en toda el área del experimento. Inmediatamente fueron efectuadas cinco lecturas por fragmento con el IMC y se registró su promedio aritmético. Según los valores medios del instrumento, los segmentos se agruparon en clases de cinco unidades de amplitud, y se abarcó todo el ámbito de lecturas. Los fragmentos agrupados constituyeron muestras de tejido foliar a las que se les determinó el contenido de $\mathrm{N}$ total mediante el método de Microkjeldahl (Henríquez et al. 1998), en el Centro de investigaciones agronómicas de la Universidad de Costa Rica. Mediante análisis de regresión lineal se estimó la función de mejor ajuste entre las lecturas del IMC y los resultados de los análisis de laboratorio. Con esta función las lecturas del IMC fueron transformadas en estimaciones del contenido relativo de $\mathrm{N}$ foliar en el arroz. Para observar valores extremos en la tonalidad de las hojas evaluadas y en las determinaciones en laboratorio de los contenidos de $\mathrm{N}$ foliar, en esta parte del estudio se consideró plantas en diferentes estados de desarrollo.

Luego de calibrar el IMC, retomando el experimento principal del estudio, para determinar la necesidad de una aplicación nitrogenada se calculó un índice de suficiencia (IS) (\%), según la ecuación: IS = ( $\% \mathrm{~N}$ foliar medio en el campo/\% $\mathrm{N}$ foliar en lotes control)*100. Los lotes control fueron áreas del arrozal donde se aplicó una dosis muy alta (el doble de la dosis normal recomendada) de $\mathrm{N}$ a las plantas para desarrollar el máximo de concentración de $\mathrm{N}$ foliar (Peterson et al. 1993).

\section{RESULTADOS Y DISCUSIÓN}

\section{Dosis de N, variantes en su fraccionamiento y ren- dimientos del arroz}

En ninguna de las combinaciones de dosis y fraccionamientos hubo diferencias significativas $(\mathrm{P} \leq$ $0,05)$ en los rendimientos del arroz por efecto del sistema de labranza (Cuadro 1). Según este resultado, después de transcurridos tres años desde la adopción de la SDR, la práctica empírica de agregar en una dosis mayor a la que se aplica en LMC no tiene fundamento científico. La cantidad adicional de fertilización nitrogenada en SDR se aplica bajo la hipótesis de que previene los síntomas de deficiencia de $\mathrm{N}$ observados en arrozales durante los primeros años luego de adoptado este sistema.

Según Sousa y Gomes (1993) las plantas de arroz irrigado cuando son cultivadas en SDR normalmente presentan al inicio de su desarrollo una deficiencia de $\mathrm{N}$. Esto se evidencia por una coloración verde amarillenta en las hojas, especialmente cuando la siembra se 
Cuadro 1. Rendimientos medios del arroz según sistemas de labranza, dosis de nitrógeno (N) y variantes en su fraccionamiento. Lote Playitas. El Pelón de la Bajura, Guanacaste, Costa Rica. Siembra en época seca 2002-2003.

\begin{tabular}{|c|c|c|c|c|c|c|c|c|}
\hline \multirow{3}{*}{$\begin{array}{c}\text { Dosis de } \\
\text { N }\end{array}$} & \multicolumn{8}{|c|}{ Variantes en el fraccionamiento de la dosis de $\mathbf{N}$} \\
\hline & \multicolumn{2}{|c|}{ A: Aplicación única } & \multicolumn{2}{|c|}{ B: $(60: 40 \%)$} & \multicolumn{2}{|c|}{$C:(40: 30: 30 \%)$} & \multicolumn{2}{|c|}{$D:(40: 20: 20: 20)$} \\
\hline & SDR & LMC & SDR & LMC & SDR & LMC & SDR & LMC \\
\hline (kg/ha) & \multicolumn{8}{|c|}{$\mathrm{kg} / \mathrm{ha}$} \\
\hline 0 & 2490 a & $2540 \mathrm{a}$ & $2790 \mathrm{a}$ & $2650 \mathrm{a}$ & $2640 \mathrm{a}$ & $2690 \mathrm{a}$ & $2653 \mathrm{a}$ & $2538 \mathrm{a}$ \\
\hline 80 & $2870 \mathrm{~b}$ & $2845 \mathrm{~b}$ & $3075 \mathrm{~b}$ & $3130 \mathrm{~b}$ & $3410 \mathrm{~b}$ & $3460 \mathrm{~b}$ & $3260 \mathrm{~b}$ & $3172 \mathrm{~b}$ \\
\hline 100 & $3220 \mathrm{c}$ & $3190 \mathrm{c}$ & $3610 \mathrm{c}$ & $3586 \mathrm{c}$ & $4215 \mathrm{c}$ & $4176 \mathrm{c}$ & $3810 \mathrm{c}$ & 3920 c \\
\hline 120 & $3705 \mathrm{~d}$ & $3680 \mathrm{~d}$ & $4215 \mathrm{~d}$ & $3865 \mathrm{~d}$ & $4740 \mathrm{~d}$ & $4635 \mathrm{~d}$ & $4640 \mathrm{~d}$ & $4586 \mathrm{~d}$ \\
\hline 180 & $4060 \mathrm{e}$ & $3988 \mathrm{e}$ & 4440 e & $4356 \mathrm{e}$ & $4870 \mathrm{~d}$ & $4785 \mathrm{~d}$ & $4810 \mathrm{~d}$ & $4872 \mathrm{~d}$ \\
\hline
\end{tabular}

Nota: Medias seguidas por la misma letra en cada columna no son significativamente diferentes $(\mathrm{P} \leq 0,05)$. En las pruebas de comparación de medias entre sistemas de labranza ninguna de las parejas de medias mostró diferencias estadísticamente significativas.

realiza sobre los rastrojos de gramíneas. Sin embargo, esta diferencia causada por la descomposición de los rastrojos tiende a desaparecer con el establecimiento de la lámina de agua en el terreno.

Los signos temporales de deficiencia de $\mathrm{N}$ posiblemente sean consecuencia de la inmovilización del $\mathrm{N}$ inorgánico, la cual es ocasionada por los microorganismos del suelo durante la descomposición de rastrojos del cultivo previo. Por tener una alta relación $\mathrm{C}: \mathrm{N}$ (60:1) los rastrojos de arroz inmovilizan parte del $\mathrm{N}$ inorgánico a corto plazo. Pero luego de varios ciclos continuos bajo SDR su acumulación en estados progresivos de descomposición puede elevar la tasa de mineralización y mantener mayor estabilidad en la dinámica total del $\mathrm{N}$ en los suelos inundados. De esta forma se podría reducir la cantidad necesaria de aplicaciones de fertilizante a largo plazo. Blevins y Frye (1993) encontraron que en SDR el contenido de materia orgánica aumentó paulatinamente con el tiempo y su más lenta mineralización conduce a formas de $\mathrm{N}$ disponible para el cultivo más sincronizadas con sus necesidades. Según Vlek y Byrnes (1986) con mejor conocimiento de los factores biológicos que controlan este proceso se puede mejorar el uso del $\mathrm{N}$ tanto de fertilizantes como el derivado de los rastrojos.

Dentro de cada una de las variantes en fraccionamiento evaluadas se presentó una relación directa altamente significativa $(\mathrm{P} \leq 0,01)$ entre la dosis de $\mathrm{N}$ y el nivel de rendimiento alcanzado en la cosecha (Figura
1). Puesto que los sistemas de labranza no afectaron los rendimientos, en este caso se incluyó datos correspondientes a la media conjunta entre SDR y LMC. Aunque en general los rendimientos aumentaron a medida que incrementó la dosis de $\mathrm{N}$, los niveles alcanzados en las variantes $\mathrm{C}$ y D fueron significativamente mayores que cualquiera en A y B. Esto significa

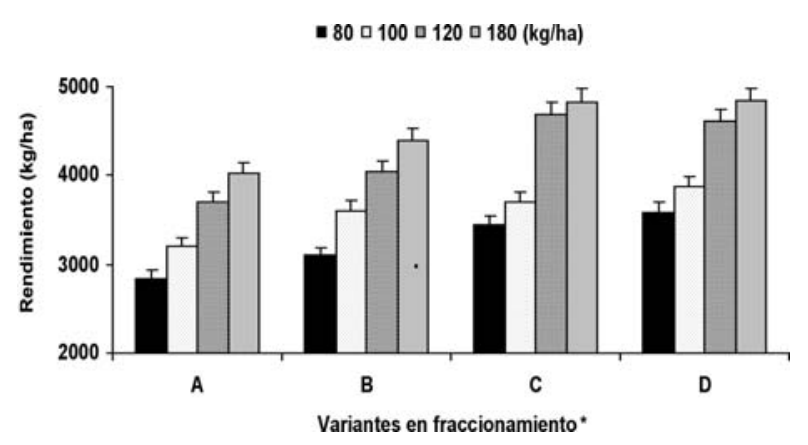

Figura 1. Relación entre dosis de $\mathrm{N}$ y rendimientos de arroz CR-1113 para las cuatro variantes en el fraccionamiento del N. Lote Playitas, Guanacaste, Costa Rica. Siembra en época seca 2002 - 2003.

\footnotetext{
* A) aplicación única de la dosis completa sobre suelo seco inmediatamente antes de aplicar la lámina de agua permanente "LAP", a $25 \mathrm{dds}$, cuando las plántulas de arroz presentaban cuatro hojas; B) $60 \%$ de la dosis al momento de aplicar la variante A y el $40 \%$ restante en la fase de iniciación del primordio de panícula "IPP", $65 \mathrm{dds}$; C) $40 \%$ en forma similar a la variante A, 30\% al IPP y $30 \%$ restante en la fase de floración "F", 85 dds; D) $40 \%$ en forma semejante a la variante A, y fracciones del $20 \%$ a 45 dds durante la fase de macollamiento "M", al IPP e IF respectivamente.
} 
que la respuesta del cultivo a dosis repartidas en tres o cuatro partes a lo largo de su ciclo de crecimiento, fue mejor que cuando el $\mathrm{N}$ se aplicó concentrado en sólo una o dos aplicaciones tempranas, probablemente como consecuencia de mayor pérdida del $\mathrm{N}$ aplicado por lixiviación y volatilización de $\mathrm{NH}_{3}$.

Lo anterior confirma la validez de los métodos tradicionales de fertilización nitrogenada, con dosis repartidas en tres o cuatro aplicaciones frente al método único de fertilización temprana sobre suelo seco. Está demostrado que altas cantidades de $\mathrm{N}$ en las primeras fases de crecimiento del arroz incrementan la suculencia de los tejidos foliares, aumentando la incidencia y severidad del ataque de plagas o enfermedades (Cartwright y Lee 1998). Esto conduce a reducciones en rendimientos por acame o volcamiento, susceptibilidad a Tagosodes oryzicolus, Rhizoctonia solani y a Pyricularia grisea, especialmente con variedades como la usada en este estudio. Investigaciones realizadas en Brasil (Sousa y Gomez 1993) mostraron que cantidades de $\mathrm{N}$ mayores a $30 \mathrm{~kg} / \mathrm{ha}$ al inicio del cultivo causaron fitotoxicidad a semillas en germinación, redujeron el número de plantas de arroz emergidas y favorecieron la incidencia de malezas. El mayor número de cariópsides vacías (\% de vaneamiento) en las variantes A $(47,5 \%)$ y B $(32,5 \%)$, con respecto a C $(26 \%)$ y D $(22 \%)$ fue una de las causas del menor rendimiento observado en las primeras, inclusive con las mayores dosis de $\mathrm{N}$ aplicado.

Los mayores niveles de rendimiento fueron alcanzados en las variantes $\mathrm{C}$ y $\mathrm{D}$ con dosis de $\mathrm{N}$ iguales o superiores a $120 \mathrm{~kg} / \mathrm{ha}$. Por tratarse de rendimientos estadísticamente no diferentes entre sí, con una media conjunta de $4.700 \mathrm{~kg} / \mathrm{ha}$, se determinó como la mejor combinación a la variante $\mathrm{C}$, con una dosis de $\mathrm{N}$ de $120 \mathrm{~kg} / \mathrm{ha}$. Otras variables complementarias en este estudio, tales como la cantidad de panículas fértiles por metro cuadrado y el número de granos llenos por panícula, presentaron alta correlación positiva $\left(\mathrm{R}^{2}=\right.$ 0,79 y 0,83 respectivamente) con el rendimiento, y sus valores máximos coincidieron con la mejor combinación elegida. Puesto que el rendimiento alcanzado sin fertilizar con $\mathrm{N}$ fue de $2.600 \mathrm{~kg} / \mathrm{ha}$, la mejor combinación (con tres fraccionamientos) permite alcanzar el máximo índice de eficiencia $(17,5 \mathrm{~kg}$ de arroz por kilogramo de fertilizante aplicado).
Uso del IMC como indicador en la fertilización nitrogenada

Las lecturas del IMC, al evaluar plantas en diversas etapas del crecimiento vegetativo, presentaron una correlación directa $\left(\mathrm{R}^{2}=0,97\right)$ altamente significativa $(\mathrm{p} \leq 0,01)$ con el contenido de $\mathrm{N}$ foliar en plantas de arroz CR-1113, determinado mediante el método de Microkjeldahl (Figura 2). Conforme cambió el color de las hojas del cultivo desde tonalidades cloróticas o amarillentas hasta verdes oscuros, las lecturas del IMC fluctuaron con valores entre 15 y 50 , correspondientes a 1,03 y $1,64 \%$ de $\mathrm{N}$ foliar. Este ámbito está incluido dentro de la media de 1,8\% indicada por Norman et al. (2003) como concentración normal de $\mathrm{N}$ en hojas de arroz. En otros estudios (Shung et al. 1999; Singh et al. 2002; Chung et al. 2000) también se encontró similar relación entre las lecturas del IMC y el contenido de clorofila en las hojas del arroz.

Las lecturas del IMC realizadas antes de la fertilización nitrogenada a lo largo de las etapas del desarrollo vegetativo mostraron una tendencia creciente, con medias generales de 31,8; 34,1 y 36,3 durante las fases de macollamiento, iniciación de panícula, y floración;

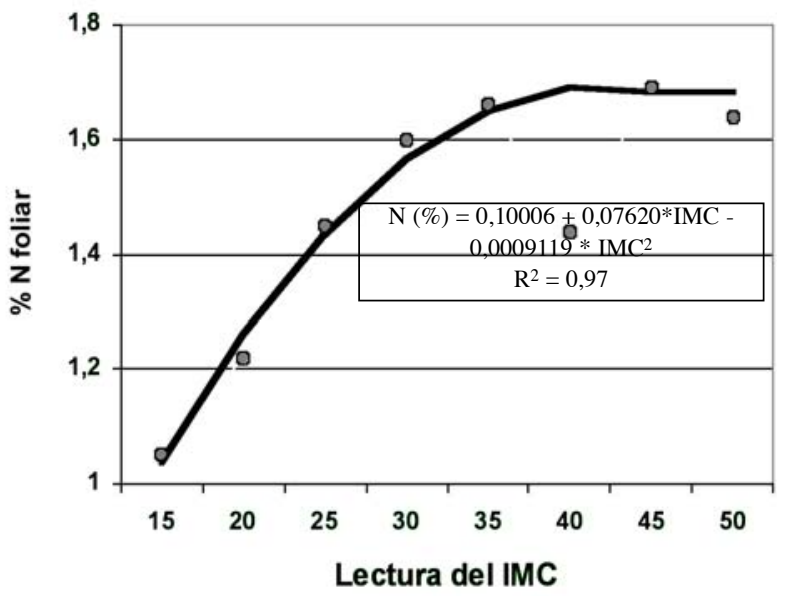

Figura 2. Relación entre lecturas del instrumento medidor de clorofila "IMC" y el contenido de nitrógeno "N" foliar en arroz variedad CR-1113.

Nota: El instrumento medidor de clorofila "IMC" usado en el estudio fue el modelo SPAD-502, desarrollado por la división especializada en productos agrícolas (Specialty products agricultural division) de la Corporación Minolta. 
Cuadro 2. Índices de suficiencia de nitrógeno foliar determinados en arroz CR-1113, según lecturas del IMC* antes de las fases del inicio de panícula y floración. Siembra de temporada seca 2002 - 2003. Lote Playitas, Guanacaste, Costa Rica.

\begin{tabular}{lccccccc}
\hline $\begin{array}{l}\text { Fase de desarrollo } \\
\text { del arroz }\end{array}$ & \multicolumn{2}{c}{ Condición en campo } & \multicolumn{2}{c}{ Parcelas control } & & \\
\cline { 2 - 5 } & $\begin{array}{c}\text { Lectura } \\
\text { IMC }\end{array}$ & $\begin{array}{c}\mathbf{N} \text { foliar } \\
\mathbf{( \% )}\end{array}$ & $\begin{array}{c}\text { Lectura } \\
\text { IMC }\end{array}$ & $\begin{array}{c}\mathbf{N} \text { foliar } \\
\mathbf{( \% )}\end{array}$ & $\begin{array}{c}\text { IS } \\
\mathbf{( \% )}\end{array}$ & Decisión \\
\hline Iniciación de panícula & 34,7 & 1,64 & 38,3 & 1,68 & 0,97 & Aplicar N \\
Floración & 36,2 & 1,66 & 38,8 & 1,68 & 0,98 & Aplicar N \\
\hline
\end{tabular}

*Nota: IMC = Instrumento medidor de clorofila Minolta SPAD-502.

equivalentes a 1,$60 ; 1,63$ y $1,66 \%$ de $\mathrm{N}$ foliar respectivamente. Durante la fase reproductiva, luego del estado de grano pastoso la tendencia fue decreciente. Sin embargo, cuando las mediciones se efectuaron cinco días después de la respectiva aplicación de $\mathrm{N}$, los resultados revelaron incrementos en la concentración de $\mathrm{N}$ foliar.

\section{IS: Índices de suficiencia de $\mathbf{N}$ y la decisión de fer- tilizar}

Los índices de suficiencia de $\mathrm{N}$ en arroz fueron determinados para decidir la necesidad de fertilización en las fases iniciación de panícula y floración (Cuadro 2). En este caso se usó como referencia el esquema de aplicaciones resultante de la mejor combinación determinada en la sección anterior del presente estudio, a saber; variante C con $120 \mathrm{~kg} / \mathrm{ha}$ de N. Las lecturas del IMC fueron tomadas antes de realizar las correspondientes fertilizaciones. Según Villar y Ortega (2002) un índice de suficiencia menor a 95\% indica deficiencia de N, y por tanto se debe realizar la fertilización prevista. En el presente ejemplo es necesario aplicar $36 \mathrm{~kg} / \mathrm{ha}$ de $\mathrm{N}$ en cada fase (30\% de $120 \mathrm{~kg} / \mathrm{ha}$ de $\mathrm{N})$.

Por la alta correlación entre los valores del IMC y el contenido de $\mathrm{N}$ foliar en las plantas obtenido mediante análisis de laboratorio, se determinó que el IMC constituye una herramienta práctica para detectar en el campo niveles críticos de $\mathrm{N}$ en las hojas. Sin embargo, se deben desarrollar métodos que permitan estimar la dosis de $\mathrm{N}$ a aplicar, cuando el valor medido sea inferior al crítico. La aplicación del IMC permite mejorar la eficiencia en el uso N, aplicando únicamente la cantidad de $\mathrm{N}$ que el cultivo requiere en los momentos propicios para optimizar su crecimiento. Esta es una forma de mejorar los rendimientos del arroz sin incrementar los gastos en la fertilización. Además, es posible reducir las pérdidas del nutrimento, evitar la contaminación de aguas subterráneas y propiciar manejos más sostenibles en los agroecosistemas con arroz inundado.

Si bien en esta investigación no se encontró evidencia de mayor requerimiento de fertilización nitrogenada en el arroz inundado bajo el sistema SDR, es conveniente realizar estudios complementarios considerando mayor amplitud de condiciones edáficas, manejos contrastantes con diferentes variedades de arroz, y mayor duración del período experimental. De esta forma se podría afinar aún más la técnica de fertilización en SDR y lograr que el cultivo aproveche los aportes de la liberación gradual del $\mathrm{N}$ procedente de la descomposición de los rastrojos, de los microorganismos del suelo y la mineralización de la materia orgánica.

\section{CONCLUSIÓN}

No se encontró suficiente evidencia para justificar que en cultivos de arroz inundado en SDR se aplique mayor cantidad de fertilizante nitrogenado que en LMC. En contraste con otras investigaciones, en el presente estudio se observó que en la variedad de arroz CR1113, la fertilización con $120 \mathrm{~kg} /$ ha de N distribuida en tres fracciones a lo largo del desarrollo del cultivo, permitió rendimientos superiores a los logrados con la dosis completa en una sola aplicación temprana. Para afinar la técnica de fertilización con N en SDR y lograr que el cultivo aproveche los aportes de la liberación gradual del $\mathrm{N}$ procedente de la descomposición de los rastrojos, de los microorganismos del suelo y la 
mineralización de la materia orgánica; es conveniente realizar estudios complementarios en los que se pueda abordar diferentes variedades de arroz, mayor amplitud de condiciones edáficas, manejos contrastantes del cultivo, y mayor duración del período experimental. Se comprobó que las lecturas del IMC permiten estimar rápidamente el contenido de $\mathrm{N}$ foliar en distintas etapas de desarrollo del cultivo. Este instrumento constituye una herramienta práctica para definir criterios objetivos en la fertilización nitrogenada del arroz. Sin embargo su uso no permite alcanzar la precisión que se logra mediante análisis foliares de laboratorio.

\section{LITERATURA CITADA}

ARREGUI, L.; MERINA, M.; MINGO-CASTEL, A. 2000. Aplicación del medidor portátil de clorofila en los programas de fertilización nitrogenada en patata de siembra. In: Pascualema J.; Ritter, E. eds. Actas del Congreso Iberoamericano de Investigación y Desarrollo en Patata. España. p.157-170.

BIRD, J.; HORWATH, W.; EAGLE, A.; KESSEL, C. 2001. Immobilization of fertilizer nitrogen in rice: effects of straw management practices. Soil Sci. Soc. Am. J. 65:1143-1152.

BLEVINS, R.; FRYE, W. 1993. Conservation tillage: an ecological approach to soil management. Adv. In Agronomy. 51: 33-78.

CARTWIGHT R.; LEE F. 1998. Management of rice diseases. Rice Production Hand Book. Arkansas University. p. 51-72.

CHUNG, S.; SUNG, J.; SUDDUTH, K.; DRUMMOND, S.; HYUN, B. 2000. Spatial variability of yield, chlorophyll content, and soil properties in a Korean rice paddy field. National Agricultural Mechanization Research Institute. Korea. 13 p.

CORDERO, A. 1993. Fertilización y nutrición mineral del arroz. EUCR, Universidad de Costa Rica., San José, Costa Rica. 100 p.

COTO-GAUCHENRAND, P. 1991. Respuesta de la variedad de arroz (Oryza sativa L.) CR-1821 y las líneas promisorias CR-8334 y CR-8341, a la fertilización nitrogenada bajo condiciones de riego. Tesis Lic. Ing. Agr. Universidad de Costa Rica, San José. 102 p.

DAVID, C.; OTSUKA, K. 1994: Modern rice technology and income distribution in Asia. Lynne Riener Publishers, Colorado, USA. 475 p.

DE DATTA, S. 1981. Principles and practices of rice production. Willey, Inc. New York, USA. 618 p.

ECK, H.; JONES, O. 1992. Soil nitrogen status as affected by tillage, crops, and crop sequences. Agron. J. 84: 660-668.

EDWARDS, W.; NORTON, L.; REDMOND, C. 1988. Characterizing macropores that affect infiltration into nontilled soil. Soil. Sci. Soc. Am. J. 52: 483-487.

HENRÍQUEZ, C.; BERTSH, F.; SALAS, R. 1998. La fertilidad de suelos, manual de laboratorio. Centro de Investigaciones Agronómicas, Universidad de Costa Rica, San José. 64 p.

JARAMILLO, S.; PULVER, E.; DUQUE, M. 2003. Efecto del manejo de la fertilización nitrogenada en arroz de riego, sobre la expresión del potencial de rendimiento en líneas élite y cultivares comerciales. (en línea). Consultado 12 nov. 2004. Disponible en: www.blanquita.com/articulos/articulo4.html

LIHONG, X.; WEIXING, C.; WEIHONG, L.; TINGBO, D.; YAN, Z. 2004. Monitoring leaf nitrogen dtatus in rice with canopy spectral reflectance. Agron. J. 96:135-142.

MOLINA, E. 2003. Características y manejo de fertilizantes que contienen Nitrógeno, Fósforo y Potasio. In : Meléndez, G.; Molina, E. eds. Fertilizantes: características y manejo. Centro de Investigaciones Agronómicas, Universidad de Costa Rica, San José. p. 37-57.

NORMAN, R.; WILSON, C.; SLATON, N. 2003. Soil fertilization and mineral nutrition in U.S. mechanized rice culture. In: Smith C.W.; Dilday R.H. eds. Rice, origin, history, technology and production. Willey, Inc., Hoboken, New Jersey. USA. p. 331-413.

NOVOA, R.; VILLAGRÁN, N. 2002. Evaluación de un instrumento medidor de clorofila en la determinación 
de niveles de nitrógeno foliar en maíz. Agricultura Técnica (Chile) 62 (1): 1-7.

PETERSON,T.; BLACKMER,T.; FRANCIS,D.; SCHEPERS, J. 1993. Using a chlorophyll meter to improve $\mathrm{N}$ management. University of Nebraska-Lincoln Cooperative Extension NebGuide G93-1171A. 4 p.

RAMIREZ, C. 2001. Nutrición nitrogenada. In: Villalobos E. ed. Fisiología de la producción de los cultivos tropicales. EUCR, Universidad de Costa Rica, San José. p. 203-224.

RANGEL-LUCIO, J.; GONZÁLEZ, G.; CASTELLANOS, R.; GARCÍA-MOYA, E.; LÓPEZ, C.; HUERTA, H. 2002. Comparación de dos pruebas para diagnosticar nitrógeno en sorgo. Terra 20: 383-390.

RICO, G.; DE DATTA, S. 1982. Efecto de fuentes métodos y época de aplicación del nitrógeno sobre el rendimiento del arroz bajo condiciones de riego. Agronomía Tropical 32(1-6): 227-237.

ROJAS, L.; MORA, A.; RODRIGUEZ, H. 2002. Efecto de la labranza mínima y la convencional en arroz (Oryza sativa L.) en la Región Huetar Norte de Costa Rica. Agronomía Mesoamericana 13(2):111-116.

SALAS, C. 2003. Nutrición mineral de plantas y el uso de fertilizantes. In: Meléndez G; Molina E.eds. Fertilizantes: características y manejo. Centro de Investigaciones Agronómicas, UCR, San José. p. 1-19.

SINGH, B.; SINGH, Y.; LADHA, J.; BRONSON, K.; BALASUBRAMANIAN, V.; SINGH, J.; KHIND, C. 2002. Chlorophyll meter - and leaf color chart bassed nitrogen management for rice and wheat in northwestern India. Agronomy Journal 94: 821-829.
SHUNG, J.; CHUNG, S.; HONG, S. 1999. Site-specific rice crop fertilizing based on chlorophyll content. In: Proceedings of 99th International Conference on Agricultural Engineering. Beijing, China. p.V-104 a V-108.

SOUSA, R.; GOMES, A. 1993. Adubacao nitrogenada em plantio directo. In: Plantio Direto de Arroz Irrigado, Uma Ponte Entre Passado e Futuro. EMBRAPA/CPATB, Brasil. p. 26 - 29.

STEVENS, G.; HEFNER, S. 1999. Use of a portable chlorophyll meter to manage crop nitrogen in rice. Agricultural publication MP729. (en línea). Consultado 12 nov. 2004. Disponible en: http:// muextension.missouri.edu/explore/miscpubs/mp0729.htm

TINARELLI, A. 1989. El arroz. Instituto Valenciano de Investigaciones Agrarias. Ed. Mundi-Prensa. Madrid. $575 \mathrm{p}$.

VILLAR, D.; ORTEGA, R. 2002. Medidor de clorofila, bases teóricas y su aplicación para la fertilización nitrogenada en cultivos. Agronomía y Forestal UC. Centro de Agricultura de Precisión de la Pontificia Universidad Católica de Chile. 8 p.

VLEK, P.; BYRNES, B. 1986. The efficacy and loss of fertilizer N in lowland rice. Fert. Res. 9:131-147.

WILSON, C.; SLATON, N.; NORMAN, R.; MILLER, D. 1998. Efficient use of fertilizer. In: Rice production hand book. University of Arkansas, Division of Agriculture, Cooperative Extension Service. Little Rock. Arkansas. U.S.A. p. 51-72.

YOSHIDA, S. 1981. Fundamentals of rice crop science. International Research Institute, Philippines. 269 p. 